\title{
Quality and Energy Aware Services Selection for IOT
}

\author{
Naresh Teja Kamatham
}

Jawaharlal Nehru Technological University Anantapuramu, Anantapur, Andhra Pradesh, India

\begin{abstract}
In recent years, Internet of Things (IoT) has received massive attention from research and industry. Integrating and composing smart objects functionalities or their services is required to create and promote more complex IoT applications with advanced featuresIn a large scale IOT environment, but the composition of thousands of entities becomes a crucial issue. In this paper, an EQSA algorithm is proposed for service composition. The proposed selection approach consists in the quality of the services in view of the satisfaction of the user through the use of a lexicographical optimization technique. In order to increase the energy conservation without decreasing the user's preferences, a most suitable service is selected among the pre candidate service ones are then Selected using the concept of relative dominance of services in the sense of Pareto. The relative dominance concept mainly depends on the energy profile and QoS attributes and users preferences.
\end{abstract}

Keywords : Internet of Things, EQSA, QoS

\section{INTRODUCTION}

The internet of things is an interconnection of various objects with their capabilities like sensing, actuation communication and computation are rapidly developed to collect the data to create the applications the users requirements

In our everyday lives, the vision of IOT is extended so number of connected devices and their innovative applications are rapidly increasing in many domains as possible.

The interconnected devices can communicate with each other through the various Communication protocols without human invocation. The IoT can be introduced in the fields of communication, e business applications. For example, a composite service would be a travel planning system that interconnects the multiple component services for flight booking, accommodation booking, car rental and itinerary planning are executed sequentially or concurrently The composition of IOT services promotes the creation of complex applications. i.e. composite services. This composite service is formed by aggregating the individual atomic services to provide new functionalities.

In this study, the term candidate service refers the invocable service, where as an abstract service is also called service classes. Each Abstract service, there may exist several concrete services which is having same functionality but different quality value. The composition plan describes how candidate services interact with each other by specifying the order of invocation.

A key issue in a composition process how effectively chooses the most relevant services that can match the user's requirements. interms of QoS, the services selection is still crucial task because dynamic and stochastic nature of IoT. In the energy model, the 
candidate services selected for a composition process which is hosted in devices are limited budget of energy. During the execution of composition plan, the services may fail due to intensive use of battery leading to unreliable of service. The mobility of devices new services are introduced but energy budget cannot be increased much more, the battery capabilities gradually increase per annum by only $5 \%$. So, finally less energy consumption services may lead to high availability of services. In this paper, an EQSA algorithm proposed for iot services composition. The main aim of this algorithm is to reduce energy consumption by slightly reducing the QoS level without affecting the user's requirements. The services selection approach is carried out by 2 methods.

\section{1) Pre-selection of services 2) Relative dominance method}

In the first phase, services selection approach is solved by using the lexicographic optimization method. This method consists of solving the sequence of each QoS attribute (availability, reliability, response time etc). The QoS attributes are ranked in the order of user preferences. For each operation, finding the candidate services providing the best quality with respect to the QoS attribute q. A threshold value of best quality is defined from the best quality value and tolerance factor $[0,1]$ represents the reduction of the quality allowed by the user.

The candidate services which do not satisfy the obtained threshold value are removed from the candidate services set. The first phase allows us reduce the search space along with selection time. The candidate services obtained in the pre selection phase are compared in the $2^{\text {nd }}$ phase $. i . e . ~ d o m i n a n c e$ concept. An utility value which represents the relative dominance of each candidate service are selected for the services composition. The utility value mainly depends on energy profile, QoS attributes, user preferences.

\section{MODELS DEFINITION}

A. Service model description.

1) concrete versus abstract services. A concrete service $(C S)$ ) is described by its functional properties in the form of transformation function Action(CSj) that takes an input Input Data(cSJ) to produce an Output $\operatorname{data}(C S j)$ and non functional attributes like $Q o S(C S j)$ and energy profile Eprof(CSj)

$$
\begin{array}{r}
c s_{j}=\left\{\operatorname{InputData}\left(c s_{j}\right), \text { OutputData }\left(c s_{j}\right), \operatorname{Action}\left(c s_{j}\right),\right. \\
\left.\operatorname{QoS}\left(c s_{j}\right), \operatorname{EProf}\left(c s_{j}\right)\right\} .
\end{array}
$$

An abstract service $A S i=\{C S 1, C S 2, \ldots \ldots . . C S n\}$ is a class of 'N'concrete services having the similar functional properties.i.e. atleast the same input data and output data but differ by the non-functional properties.

\section{2)Abstract versus Concrete Composite Service: An} abstract composite service is represented by the aggregation of abstract services(services classes) with out representing the concrete services. A concrete composite service is example of an abstract composite service obtained by mapping the each abstract service (e.g., localization of an object) to one of its concrete services (e.g., RFID-based localization, vision-based localization).

\section{B.Energy Profile Model:}

The energy profile Eprof(CSj) of a concrete service is descibed by following parameters like $E \operatorname{cost}(C S J)$ and AutoS(CSj). The Ecost $(\mathrm{CSj})$ represents the overall energy consumption during the execution of the service. The AutoS(CSj) represents residual Energy level of the device which hosting the service.

$$
\begin{aligned}
& \text { AutoS }\left(c s_{j}\right) \\
& \quad=\text { EnergyDevice }\left(c s_{j}\right)-\text { EnergyThreshold }\left(c s_{j}\right)
\end{aligned}
$$


Energy device and Energy threshold represented as, the charge level of battery powered device hosting a service and threshold value under which device cannot provide a service anymore.

$$
\operatorname{ECost}\left(c s_{j}\right)=\operatorname{ConsRate}\left(c s_{j}\right) \cdot \operatorname{ExecTime}\left(c s_{j}\right)
$$

ConsRate represents the consumption rate and Exec Time (CSj) represents the execution time of the service.

Finally, the energy profile of a service is defined as ratio of its energy cost and its autonomy is represented as

$$
\operatorname{EProf}\left(c s_{j}\right)=\frac{\operatorname{ECost}\left(c s_{j}\right)}{\operatorname{AutoS}\left(c s_{j}\right)}
$$

\section{1) QoS Level Based Services Pre-selection:}

The Qos aware services selection can be done by using the Lexicographic optimization. In this technique, ranking the each QoS attribute in an order based on the user's preferences. Let us consider 6 candidate services $\mathrm{C} 1, \mathrm{C} 2, \mathrm{C} 3, \mathrm{C} 4, \mathrm{C} 5, \mathrm{C} 6$.

$\mathrm{C} 1=\{\mathrm{EP}=0.05, \mathrm{cost}=10.3, \mathrm{RT}=20, \mathrm{Av}=90\}$

$\mathrm{C} 2=\{\mathrm{EP}=0.5$, cost $=9, \mathrm{RT}=21, \mathrm{Av}=92\}$

$\mathrm{C} 3=\{\mathrm{EP}=0.7, \mathrm{cost}=10, \mathrm{RT}=22, \mathrm{Av}=91\}$

$\mathrm{C} 4=\{\mathrm{EP}=0.15, \mathrm{cost}=13, \mathrm{RT}=18, \mathrm{Av}=93\}$

$\mathrm{C} 5=\{\mathrm{EP}=0.15, \mathrm{cost}=8, \mathrm{RT}=27, \mathrm{Av}=95\}$

$\mathrm{C} 6=\{\mathrm{EP}=0.1, \mathrm{cost}=9.5, \mathrm{RT}=25, \mathrm{Av}=85\}$

The user selects cost is his first priority. So, from the above 6 candidate services cost $=90$ is the best quality of service.

Here, we define a threshold value and tolerance factor which is characterizing the decrease of quality allowed by the user.

$$
\begin{aligned}
& \operatorname{qos}_{q}^{\text {Threshold }}= \begin{cases}q o s_{q}^{*}-q o s_{q}^{\text {Decrease }}, & \text { if } q \text { is positive } \\
q o s_{q}^{*}+q o s_{q}^{\text {Decrease }} & \text { otherwise }\end{cases} \\
& \text { with } \quad q^{\text {os }}{ }_{q}^{\text {Decrease }}=q o s_{q}^{*} \cdot \delta_{q}
\end{aligned}
$$

Where qos*q, qosdecrease, del $\mathrm{q}$ represents best quality value, decrease the quality which allowed by the user, tolerance factor ranging from $[0,1]$.

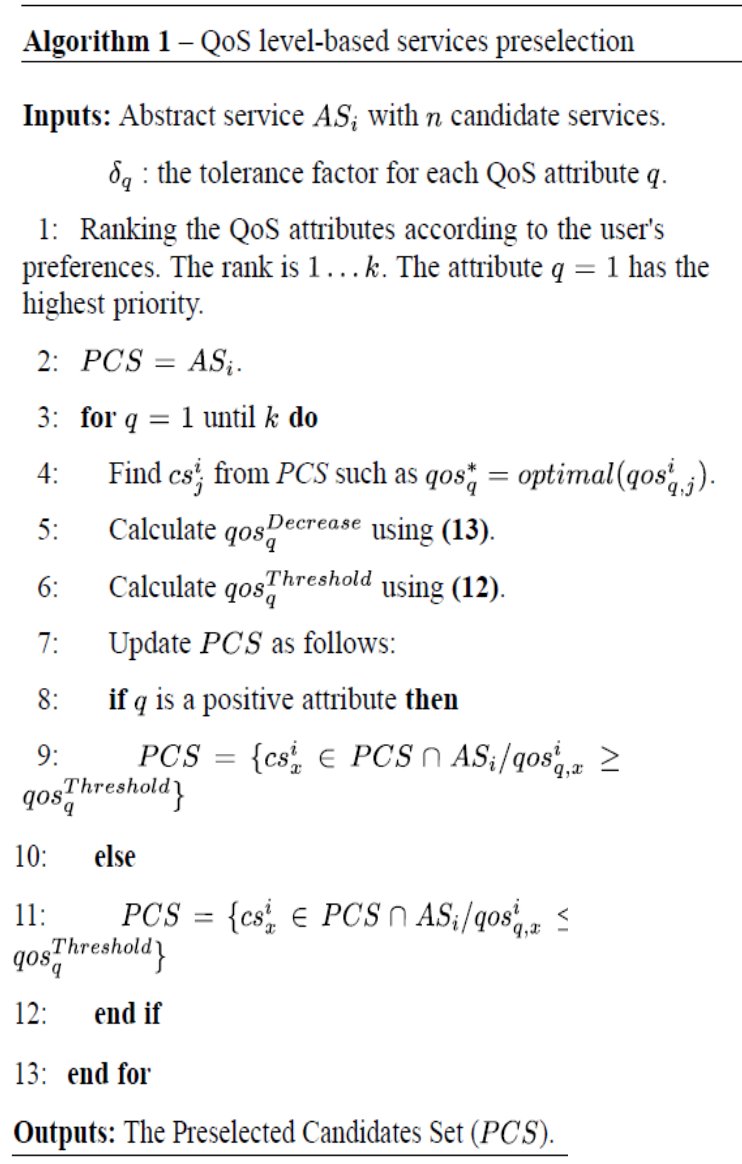

\section{2) Relative dominance based services selection:}

In this phase, the pre selected candidate services are compared using the value which reflects their relative dominance. The dominance value mainly depends on its energy profile, QoS attributes and users preferences. Finally, the best services in terms of relative dominance are selected for the composition process. 
The candidate services which are selected for PCS (CS) are given below

$$
\begin{aligned}
& \mathrm{C} 1=\{\mathrm{EP}=0.05, \text { cost }=10.3, \mathrm{RT}=20, \mathrm{Av}=90\} \\
& \mathrm{C} 2=\{\mathrm{EP}=0.5, \text { cost }=9, \mathrm{RT}=21, \mathrm{Av}=92\} \\
& \mathrm{C} 3=\{\mathrm{EP}=0.7, \text { cost }=10, \mathrm{RT}=22, \mathrm{Av}=91\}
\end{aligned}
$$

\begin{tabular}{|l|l|l|l|l|l|}
\hline C.S & E.P & COST & R.T & Av & R.D \\
\hline 1$)$ & 3 & 1 & 3 & 1 & 3.6 \\
\hline 2$)$ & 2 & 3 & 2 & 3 & 5.4 \\
\hline 3$)$ & 1 & 2 & 1 & 2 & 3.4 \\
\hline
\end{tabular}

Fig 1. EP and QoS dominances to calculate

\section{2) Relative Dominance:}

Let Eprof (cs1)=Ecost(cs1)/AutoS(cs1) and Eprof(cs2)=Ecost(cs2)/AutoS(cs2) be the energy profile of CS1 and CS2.

\section{Definition1:}

QoS Attribute based Dominance: It can be represented as how many number of candidate services are dominated with respect to its attributes like 'COST'. From the above candidate services CS1 dominated by remaining 2 services. so its value is Dom(cs1)=3. Remaining also follow the same rule.

\section{Definition2:}

\section{Energy profile based Dominance:}

In the energy model description, The candidate service providing the lowest

energy profile value will have the highest energy profile-based dominance which is equal to the value 3 .

Algorithm 2 - Relative dominance-based services selection

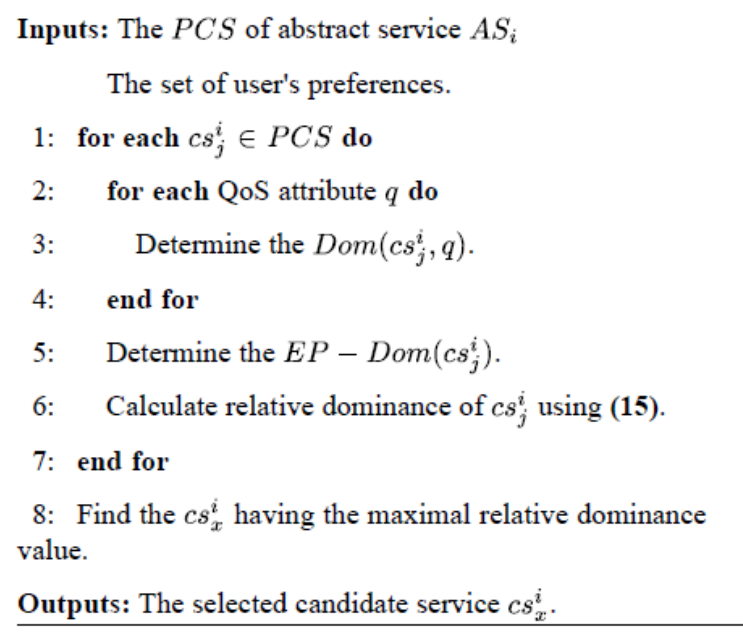

Relative dominance

The value which is having the highest relative dominance is selected for the service composition.

The Energy model is described below for how to calculate energy profile of a candidate service and then energy consumption. The battery of each device has initial charge value "Cintial" chosen randomly in the interval [0.7Cmax to $1.0 \mathrm{Cmax}$. Where the Cmax represents the maximum battery charge. When a service is requested, a charge chosen randomly in the interval [100mA.S, $10000 \mathrm{~mA} . \mathrm{s}]$ is subtracted from the actual battery charge of the device hosting the service. Cmax=1500 mA.h

Cthreshold $=30 \%$ of $\mathrm{Cmax}$

A device can stop providing a service when the battery level reaches its threshold level and the service considered to be failed in the selection view. in the proposed selection approach, the added value can be compared by using the 2 algorithms.

1) EQSA: Here both energy profile and quality value can be considered for the services selection

2) EQSA-EP: Here the only the energy profile values can be considered i.e. lower energy profile services are selected.

3) EQSA-QoS: Here the only quality attributes are considered for selection and composition process. 


\section{RESULTS AND DISCUSSION}

Simulation Results:

- Relative Dominance: it is sum of dominances of all the candidate services.

- Energy Consumption: it is sum of the energy consumed by individual components.

- Composition life time: The number of compositions are executed before the first concrete service failure. A service is failure when its residual energy no longer sufficient to be used.

1) Relative dominance Vs. no. of concrete services :

The relative dominance of a EQSA algorithm allows to selecting candidate services maximizing both EPbased \& QoS-based dominance but in EQSA-QoS algorithm allows only considering QoS values alone, which leads to reduce the relative dominance.

\section{Relative dominance versus number of concrete services}

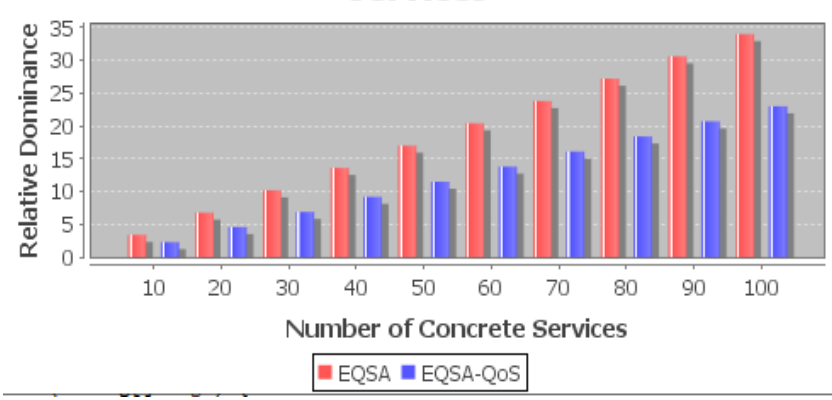

2) Energy consumption Vs No. of Tasks:

The figure shows that the energy consumption of the composite path(EQSA) increases when the number of service classes(Tasks) increases. In fact, increasing the size of composite path will increase the number of selected services which causes more power consumption.

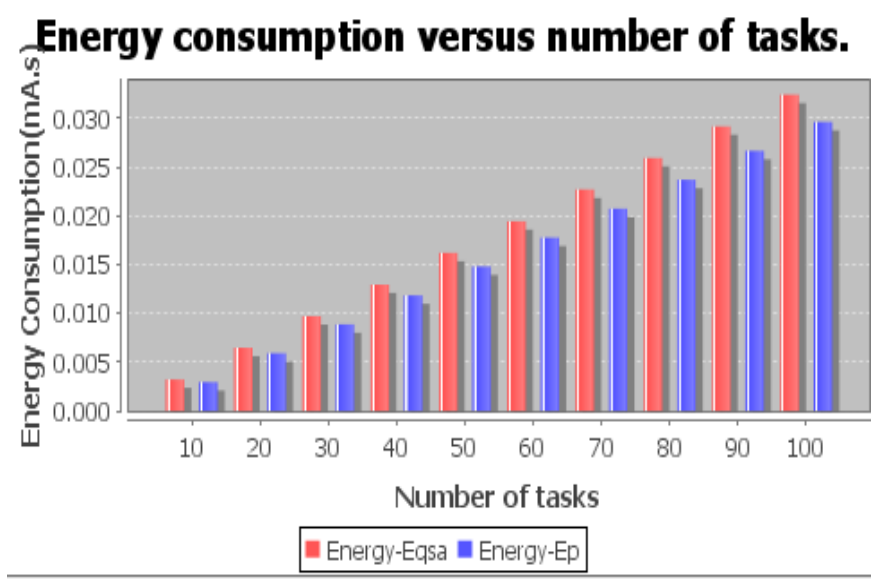

3) Composition life time Vs no. of concrete services:

Composition life time=Total initial energy $/$ energy per sec.

The composition lifetime of EQSA algorithm is more efficient than the EQSA-QoS. Because EQSA-QoS select the services with high energy profile which leads to reduce the composition life time

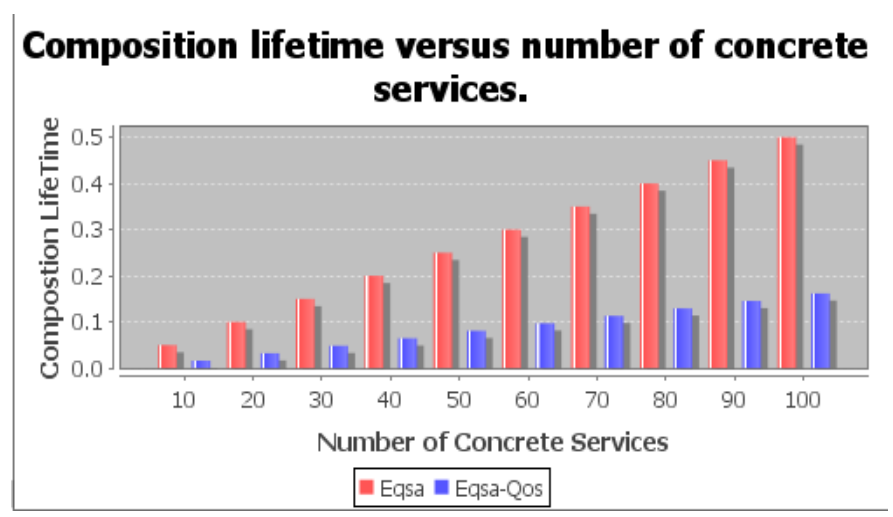

\section{IV.CONCLUSION AND PERSPECTIVES}

In this paper, QOS aware services selection with reduced energy consumption approach for IoT environment is presented. It can be solved as a biobjective optimization problem includes quality value and minimization of energy consumption which provides the high availability of services along with users satisfaction. A QoS based services selection can 
be achieved by using the Lexicographic optimization technique. In the next phase, a relative dominance concept is used for those candidate services which are Pareto equivalents. The pre selected candidate services are compared for best service selection and the relative dominance value can be calculated from the energy profile and QoS attributes. The posted simulation results promising performances of the proposed EQSA algorithm.

\section{REFERENCES}

[1]. Y. Ngoko, A. Goldman, and D.Milojicic, "Service selection in web service compositions optimizing energy consumption and service response time," J. Internet Services and Applicat., vol. 4, no. 1, pp. 1-12, Dec. 2013.

[2]. D. Ardagna and B. Pernici, "Adaptive service composition in flexible processes," IEEE Trans. Softw. Eng., vol. 33, no. 6, pp. 369-384, Jun. 2007.

[3]. T. Yu, Y. Zhang, and K.-J. Lin, "Efficient algorithms for Web services selection with end-to-end QoS constraints," ACM Trans. Web, vol. 1 no. 1, pp. 6:1-6:26, May 2007.

[4]. G. A. Garcia Llinas and R. Nagi, "Network and QoS-based selection of complementary services," IEEE Trans. Services Computing, vol. 8, no. 1, pp. 79-91, Feb. 2015.

[5]. Q.Wu and Q.Zhu, "Transactional andQoSaware dynamic service composition based on ant colony optimization," Future Generation Comput. Syst., vol. 29, no. 5, pp. 1112-1119, Jul. 2013.

[6]. M. Alrifai, T. Risse, and W. Nejdl, "A hybrid approach for efficient Web service composition with end-to-end QoS constraints," ACM Trans. Web, vol. 6, no. 2, pp. 7:1-7:31, May 2012.
[7]. S. X. Sun and J. Zhao, "A decomposition-based approach for service composition with global QoS guarantees," Inf. Sci., vol. 199, pp. 138153, Sep. 2012.

[8]. Furthmüller and O. P. Waldhorst, "Energyaware resource sharing with mobile devices," Comput. Networks, vol. 56, no. 7, pp. 19201934, May 2012.

\section{Cite this article as :}

Naresh Teja Kamatham, "Quality and Energy Aware Services Selection for IOT", International Journal of Scientific Research in Science and Technology (IJSRST), Online ISSN : 2395-602X, Print ISSN : 23956011, Volume 7 Issue 1, pp. 93-98, January-February 2020. Available at doi : https://doi.org/10.32628/IJSRST207126 Journal URL : http://ijsrst.com/IJSRST207126 